\title{
http://ensaios.usf.edu.br/
}

\section{CUIDANDO DOS CUIDADORES: UM OLHAR SOBRE A DEPRESSÃO EM CUIDADORES DE IDOSOS}

\author{
CARING FOR CAREGIVERS: A LOOK AT DEPRESSION IN CAREGIVERS OF ELDERLY
}

\author{
CASTANHASSI, Priscila ${ }^{1}$; PIOVEZAN, Nayane Martoni ${ }^{1}$; MUNER, Luana Comito²; \\ BATISTA Helder Henrique Viana ${ }^{1}$ \\ ${ }^{1}$ Universidade São Francisco, ${ }^{2}$ Faculdade Cathedral \\ priscila.castanhassi@gmail.com
}

\begin{abstract}
RESUMO. A depressão, atualmente um dos transtornos com maior prevalência na população, caracteriza-se pela perda de interesse ou prazer pelas atividades, humor deprimido, causando prejuízo no funcionamento social, profissional e em outras áreas relevantes na vida de um indivíduo. Em específico, na vida do cuidador pode causar sofrimento, angústia, ansiedade e alterar a rotina, atividades e convívio social, gerando possíveis sintomas depressivos. O presente estudo teve por objetivo rastrear a sintomatologia depressiva em cuidadores informais de idosos com limitações para a realização de atividades de vida diária, residentes na Região Metropolitana de Campinas-SP. Participaram 46 cuidadores com idades entre 22 e 79 anos $(M=46,89 ; D P=13,91)$ e a maioria $76,1 \%(n=35)$ do sexo feminino, sendo que $50 \%$ dos cuidadores $(n=23)$ dedicava mais de nove horas diárias de cuidados ao idoso. Utilizou-se o Inventário Sociodemográfico do Cuidador Informal e a Escala Baptista de Depressão. Os cuidadores que residem com os idosos apresentaram maior sintomatologia depressiva quando comparados com aqueles que não residem junto ao idoso. Verificou-se diferença significativa para a variável situação profissional, com maior presença de sintomas no grupo de cuidadores desempregados e menor entre os aposentados e os empregados. Neste estudo, a sintomatologia depressiva foi menor do que os resultados encontrados em outras pesquisas nacionais. Ao se conhecer com mais profundidade o contexto estudado poderá ser possível o preparo adequado dos cuidadores para o desempenho de suas tarefas e principalmente, minimizar os danos causados sobre si mesmos.
\end{abstract}

Palavras-chave: sintomas depressivos, sobrecarga emocional, apoio ao cuidador.

ABSTRACT. Depression, currently one of the most prevalent disorders in the population, is characterized by loss of interest or pleasure in activities, depressed mood, causing impairment in social, professional, and other relevant areas of life in an individual. Specifically, in the life of the caregiver can cause suffering, distress, anxiety and change the routine, activities and social life, generating possible depressive symptoms. The present study aimed to track the depressive symptomatology in informal caregivers of the elderly with limitations to perform daily life activities, living in the Metropolitan Region of Campinas-SP. A total of 46 caregivers aged 22-79 years $(M=46.89, S D=13.91)$ and most of them $76.1 \%(n=35)$ were women, $50 \%$ of caregivers $(n=23)$ spent more than nine hours a day caring for the elderly. The Sociodemographic Inventory of the Informal Caregiver and the Baptist Depression Scale were used. Caregivers living with the elderly presented greater depressive symptomatology when compared to those who did not live with the elderly. There was a significant difference for the professional status variable, with a greater presence of symptoms in the group of unemployed caregivers and lower among retirees and employees. In this study, the depressive symptomatology was lower than the results found in other national surveys. By knowing more 
deeply the context studied, it may be possible to prepare the caregivers for the performance of their tasks and, especially, to minimize the damage caused to them..

Keywords: depressive symptoms, emotional overload, caregiver support.

\section{INTRODUÇÃO}

Tendo em vista a importância do bem-estar do cuidador de idosos, cuja expectativa de vida tem aumentado nos últimos anos e, consequentemente a possibilidade de ser acometido por doenças de ordem diversas, diminuindo sua independência e autonomia, em que há a necessidade de acompanhamento de um cuidador (SILVA, 2005; BODSTEIN; LIMA; BARROS, 2014), optou-se pelo desenvolvimento deste trabalho a fim de rastrear a sintomatologia depressiva em cuidadores informais de idosos com doenças que limitem a vida diária.

De acordo com o critério estabelecido pela Organização Mundial da Saúde (OMS, 2005) e adotado pelo Brasil, deve-se utilizar a idade de referência de 60 anos para se referir ao sujeito idoso, participante direto ou não de levantamentos demográficos, embora não somente a idade cronológica deva ser considerada para definir um indivíduo como idoso (SILVA, 2005). Segundo Silva (2005) o Brasil está passando por um aumento significativo na população de idosos. De acordo com estimativas do Instituto Brasileiro de Geografia e Estatística (IBGE) em 2025, da população brasileira será de aproximadamente 32 milhões de pessoas acima de 60 anos (BODSTEIN, LIMA \& BARROS, 2014). Para Pessanha (2013) um dos motivos apresentados refere-se à transformação social do papel da mulher na sociedade brasileira nas últimas décadas, que ocasionou um declínio na fecundidade de 5,8 filhos em 1960 para 1,9 filhos em 2010.

Embora a expectativa de vida tenha apresentado elevação contínua, não se percebe nenhuma estruturação efetiva de políticas públicas tanto para os idosos quanto para seus cuidadores, objeto principal deste trabalho. Desta forma, torna-se necessário investigar a sobrecarga psíquica na vida do cuidador a fim de se levantar questionamentos e elaborar propostas para a promoção da integração, saúde e bem-estar psicológico desta parcela da população (CRUZ; LECHETA; WACHHOLZ, 2009).

Além do envelhecimento demográfico, Campos (2004, apud DIAS JUNIOR; COSTA; LACERDA, 2006) destaca também um aumento na longevidade dos idosos que se deve, principalmente, aos avanços tecnológicos relacionados à área da saúde que ocorreram ao longo dos anos, como as vacinas, antibióticos, quimioterápicos entre outros. Entretanto, a sociedade atual não está preparada para essa alteração no perfil da população (MENDES et al., 2005).

Há ainda o fato de se viver em uma economia capitalista na qual a produtividade do indivíduo é fortemente valorizada. Neste sentido, os idosos podem ser marginalizados por não participar da vida produtiva ao se aposentar e sentirem-se desvalorizados pela sociedade, podendo ocorrer um declínio de autoestima e o isolamento social. Enfrentam também, uma redução na renda, ocasionado pelo valor da aposentadoria, o que tende a afetar sua qualidade de vida (MENDES et al. 2005).

Para Cruz, Lecheta e Waccholz (2009), quando o idoso se aposenta os papéis se invertem na família, ele perde seu papel de comando e geralmente são os filhos que passam a assumir a responsabilidade pelos pais. Muitas vezes o relacionamento com a família pode ser difícil devido à falta de compreensão com o idoso, que se torna cada vez mais dependente de seu cuidador. Os autores ainda ressaltam que a existência de conflitos familiares anteriores ao estado de dependência, o ato de cuidar pode tornar-se mais desgastante.

Sommerhalder (2001) salienta que o poder de decisão e da participação familiar está relacionado ao nível de dependência física, cognitiva e econômica do idoso. Por exemplo, os 
casos em que os idosos moram em casas de filhos e/ ou outros cuidadores e precisam constantemente de apoio para a realização de atividades de vida diária. Mendes, et al. (2005) consideram que a família e comunidade são muito importantes na construção de uma nova estrutura para o idoso e também para proporcionar novas opções para aqueles que deixaram de trabalhar. Segundo Tomomitsu, Perracini e Neri (2013) há uma obrigatoriedade moral para que a família cuide do idoso.

Os cuidadores podem ser tanto formais (profissionais remunerados pelo trabalho de cuidar do idoso) ou informais (executado por familiares, amigos, entre outros que não são remunerados pelo ato de cuidar). Esta é uma atividade reconhecida pelo Ministério do Trabalho e Emprego, em que o descreve como "acompanhante de idosos, cuidador de pessoas idosas e dependentes, cuidador de idosos domiciliar, cuidador de idosos institucional, gerosister". Ainda, segundo a Classificação Brasileira de Ocupações - CBO 2002 (MTE, 2002) para o exercício da atividade de cuidadores a idade mínima é de 18 anos, além de ter concluído o ensino fundamental e participar de cursos livres com carga horária acima de 80 horas. Podem trabalhar em instituições particulares, públicas, ONG's e também nos domicílios dos idosos atendidos. As atividades, de forma detalhada, envolvem o cuidado com a higiene e aparência, auxilio nas atividades diárias, estar atento à todas as atividades, comportamentos e sinais dados pelo idoso, acompanhar durante consultas e exames médicos, observar sinais vitais, entre outros.

Já os cuidadores informais são pessoas integrantes da mesma família que o idoso, vizinhos ou que tenham algum tipo de relação de afinidade que oferecem assistência e auxílio para a realização de atividades de vida diária para os indivíduos que apresentam nível parcial ou total de dependência (TOMOMITSU; PERRACINI; NERI, 2013). De acordo com Neri (2007) e Pessanha (2013), a maioria dos cuidadores informais de idosos é do sexo feminino, cônjuges e filhas dos idosos.

Observa-se que um dos familiares é geralmente considerado o cuidador principal, responsável pelos cuidados básicos, tais como, a alimentação, administração de medicamentos, acompanhamento médico, higiene pessoal, entre outros cuidados dispensados ao idoso debilitado que apresenta alterações cognitivas, doenças mentais e doenças físicas moderadas a severas (Sommerhalder, 2001). As responsabilidades pelo idoso faz com que o cuidador seja exposto a uma série de situações estressantes que, por diversas vezes, são realizadas sem informação e falta de apoio psicossocial adequados para o cuidado necessário (STACKFLETH et al. 2012).

Segundo Cruz, Lecheta e Wachholz (2009) há uma sobrecarga física e emocional sobre os cuidadores, pois assumem uma tarefa estressante, desgastante e que cujo o vínculo familiar possivelmente indica uma ligação afetiva com o idoso. Para os autores, muitas vezes são pessoas da própria família que assumem esta função sem que tenham o conhecimento necessário e o suporte adequado para tanto.

Diante do aumento da expectativa de vida e da progressão de doenças crônicas o papel do cuidador é essencial para a qualidade de vida do idoso dependente (PESSANHA, 2013). Tomomitsu, Perracini e Neri (2013) afirmam que há grande probabilidade de que o cuidador também seja idoso, portador de doença crônica e outras incapacidades decorrentes da idade. Desse modo, o sentimento de sobrecarga, principalmente de idosos com déficit cognitivo, tende a gerar comprometimento físico, ansiedade e depressão nos cuidadores (GARRIDO; ALMEIDA, 1999 apud PESSANHA, 2013). De acordo com Piquart e Sörensen (2006; 2007, apud TOMOMITSU; PERRACINI; NERI, 2013) os cuidadores idosos apresentam menor satisfação com a vida, mais depressão e mais problemas de saúde.

O envolvimento afetivo e todas as mudanças geradas na vida do cuidador, bem como deixar de lado seu próprio projeto de vida em detrimento do idoso, a diminuição de tempo para relacionar-se com amigos, sobrecarga física e frustrações diversas podem aumentar o nível de estresses no cuidador (STACKFLETH et al., 2012). Gil e Bertuzzi (2006) relatam a 
necessidade de haver suporte e apoio para o cuidador, já que este poderá abdicar de suas próprias necessidades e responsabilidades para acompanhar e auxiliar o idoso na realização das atividades de vida diária e, em decorrência disso, tende a se identificar com angústias e conflitos existentes na pessoa do idoso e passa a dedicar-lhe muito mais cuidado e atenção mesmo quando não há recursos materiais e psicológicos para fazê-lo o que acarreta mais angústia àquela já existente.

A tarefa de cuidar do idoso dependente, cuja demanda de assistência cresce com o passar do tempo, tende a ser o gatilho para o início da sintomatologia depressiva (CRUZ; LECHETA; WACHHOLZ, 2009). Os autores salientam que os cuidadores têm de duas a três vezes mais chance de desenvolver transtornos depressivos quando comparados a não cuidadores. Gaioli, Furegato e Santos (2012) consideram que a dedicação aos cuidados do idoso dependente ao longo do tempo pode causar comprometimentos à saúde do cuidador, levando a quadros de ansiedade, depressão e distúrbios de comportamento.

É preciso considerar conjuntamente a questão da depressão dos idosos dependentes. Embora a incidência de depressão não seja atrelada ao aumento da idade (PAPALIA; OLDS; FELDMAN, 2006) tal situação tende a ser mais profunda em idade avançada do que em outras etapas da vida (BROMLEY, 1990 apud BAPTISTA et al., 2006). Mesmo havendo grande diversidade entre a população idosa no país, as diversas perdas, os eventos estressantes e as alterações fisiológicas naturais da idade desempenham um forte papel na ocorrência de transtorno de humor em idosos. Com o aumento da idade, o indivíduo precisa lidar com o luto de grandes perdas em sua vida, como o falecimento de entes queridos, afastamento do emprego e de atividades sociais e problemas relacionados à própria saúde (BAPTISTA et al., 2006).

Para Ferreira, Lima e Zerbinatti (2012), os principais fatores de variação na terceira idade são: declínio cognitivo, história de depressão anterior, condições socioeconômicas desfavoráveis e comorbidades psiquiátricas. Também devem ser considerados os processos de degeneração cerebral, processos genéticos e a baixa autoestima, quando se consideram um peso para sua família (COUTINHO et al., 2003).

De acordo com Baptista (2004), a depressão causa sofrimento tanto para o paciente quanto para os indivíduos que convivem com ele, alterando a rotina, atividades e convívio social. No caso de paciente idoso, esta convivência tende a ser maior com seus os cuidadores, que acabam por se colocar em segundo plano dirigindo seus esforços totalmente para os idosos e tal situação tende a gerar sentimentos ambíguos em relação ao paciente, bem como insegurança e medo (PESSANHA, 2013). Maiores níveis de estresse, ansiedade e depressão em pessoas cuidadores de idosos foram encontrados quando comparados às normas populacionais, sendo associado a doenças de âmbito físico e à diminuição do bem-estar e qualidade de vida (PEREIRA; CARVALHO, 2012; PESSANHA, 2013).

A depressão é caracterizada pela perda de interesse ou prazer pelas atividades, humor deprimido, causando prejuízo no funcionamento social, profissional e em outras áreas relevantes na vida de um indivíduo. A característica comum desses transtornos é a presença de humor triste, vazio ou irritável, acompanhado de alterações somáticas e cognitivas que afetam significativamente a capacidade de funcionamento do indivíduo. (APA, 2014).

As mulheres, geralmente, são as principais cuidadoras, em princípio as filhas ou noras mais velhas, seguidas das filhas solteiras (SOMMERHALDER, 2001). No contex to familiar, as filhas se responsabilizam por atividades voltadas às tarefas de higiene, uso de medicamentos, tarefas do lar, enquanto os filhos são voltados a questões financeiras e de tarefas externas, como levar o idoso ao médico.

Lahan (2003, apud PESSANHA, 2013) ressalta que este tipo de relação apresenta certo grau de afeto entre o idoso e o cuidador, que em geral estão presentes sentimentos como amor, senso de obrigação ou de dívida e piedade. Ainda, no cuidado podem se manifestar expressões de espiritualidade, encontro de um sentido maior na vida e um equilíbrio 
biopsicossocial (ALMEIDA et al., 2013; ROCHA; VIEIRA; SENA, 2008). Silveira, Caldas e Carneiro (2006) também encontraram em suas pesquisas, raiva, paciência, tristeza, revolta, pena, medo de ficar doente, medo de o idoso sofrer, medo da morte do idoso, entre outros com forte conotação emocional. Sommerhalder (2001) reforça a presença do sentimento de culpa, principalmente entre as mulheres devido à pressão sofrida pela sociedade de se cumprir o papel de cuidadora, mesmo não sendo esta a sua própria vontade e capacidade.

Ao longo dos anos tem-se realizado estudos sobre os aspectos psicológicos dos cuidadores de idosos. Pawlowski et al., (2010) avaliaram a presença de sintomas depressivos em cuidadores de familiares com síndrome demencial comparados a não cuidadores de diferentes faixas etárias e avaliar a presença de sintomas depressivos em cuidadores com diferentes graus de parentesco com a pessoa cuidada. A pesquisa foi realizada com 84 cuidadores e 101 não cuidadores residentes na Região Metropolitana de Porto Alegre-RS. Os participantes responderam um Inventário Sociodemográfico e o Inventário Beck de Depressão para avaliar a relação entre sintomas depressivos de ambos os grupos de cuidadores e de não cuidadores. Foram encontrados níveis altos de depressão em indivíduos da faixa etária (entre 56 e 64 anos de idade) e níveis mais elevados de sintomatologia depressiva e com maior gravidade nos aspectos cognitivos e afetivos do Inventário Beck de cuidadores quando comparados aos participantes não cuidadores.

Sommerhalder (2001) objetivou descrever as avaliações cognitivas positivas e negativas informadas por cuidadores. O estudo avaliou as descrições de 20 cuidadores, que foram submetidos a uma entrevista em profundidade e responderam ao Inventário de Benefício e Ônus Percebido. Os resultados sugeriram que há complexidade entre o que é percebido como ônus e bônus por parte dos participantes. Os benefícios psicológicos foram os mais citados, com predominância para sentimentos de responsabilidade, utilidade e fortaleza (100\%); novo significado para a vida, satisfação em cuidar, cuidar por amor (95\%); mudança na crença da relação velhice/dependência, proximidade e sentir-se bem consigo (90\%); cumprimento do dever cristão, reconhecimento do idoso (85\%). Entre os benefícios sociais informados apareceram: valorização social e dar exemplo de solidariedade (70\%); melhoria de relações familiares $(60 \%)$. Avaliação negativa no domínio psicológico esteve associada a sentimentos de compaixão pelo idoso e tristeza pela irreversibilidade de sua doença (80\%); rejeição familiar da condição do idoso (65\%); falta de privacidade e irritação (60\%); ansiedade e tristeza (55\%). No domínio social, as avaliações negativas relacionaram-se a falta de tempo para a família e amigos $(75 \%)$ e falta de tempo para o lazer $(70 \%)$. O ônus físico foi relatado por: cansaço físico (75\%); falta de tempo para descansar $(55 \%)$; piora no estado de saúde $(55 \%)$ e insônia (50\%). As estratégias individuais de enfrentamento, bem como o suporte familiar foram relacionados positivamente com as avaliações positivas e com o bemestar subjetivo.

Pessanha (2013) analisou a presença de sobrecarga emocional e física e seus impactos em cuidadores de idosos com demência, relacionando-as ao cuidado prestado por estes cuidadores aos pacientes por eles cuidados. Os instrumentos utilizados foram a Ficha de Caracterização Sociodemográfica para o cuidador informal e outra para o idoso e entrevista semiestruturada. Participaram 46 cuidadores, sendo 5 homens e 41 mulheres, sendo cônjuges, filhos (as), netos, genros/ noras, vizinhos e amigos dos idosos. Os resultados encontrados quanto à questão sociodemográfica indicaram que a maioria dos cuidadores são do sexo feminino (80\%), adultos $(90 \%)$ e que residem com os idosos $(90 \%)$. Quanto à questão familiar, tem-se cônjuge (60\%), filhos (30\%) e netos (10\%). O nível de escolaridade dos cuidadores encontrado foi que $50 \%$ concluíram o ensino médio e $30 \%$ concluíram o ensino fundamental. Por fim, a autora entende que ser cuidador demanda de muitos sacrifícios e perdas graduais (financeiros, psicológicos e de tempo, etc.) diante da situação de dependência exigida pelo idoso. 
O objetivo da pesquisa de Silveira, Caldas e Carneiro (2006) foi de contribuir para uma melhor compreensão dos cuidadores informais de idosos altamente dependentes. Os dados foram colhidos através de entrevistas e em trabalhos em grupo com 24 cuidadores. Utilizou-se o método de análise de conteúdo sobre os temas que emergiram nesses grupos, sendo: negação versus aceitação da doença antes e depois do diagnóstico, como e por que surge o cuidador principal, características do cuidador, significados do ato de cuidar, história do relacionamento do cuidador com a pessoa cuidada antes da doença, relacionamento cuidador/ idoso após a doença, mudanças na vida do cuidador, mudanças na família, causas para o surgimento da doença. Os resultados sugerem que existem três dimensões para as questões estudadas, ou seja, há implicações dos legados, das transmissões multigeracionais, das repetições dos padrões, dos mitos e das crenças características de cada sistema familiar; o idoso também é um participante ativo na decisão de quem vai cuidar dele; o grupo de suporte é um recurso muito importante para os familiares.

Gratao et al. (2012) realizaram uma pesquisa epidemiológica, descritiva e transversal, para descrever a sobrecarga e o desconforto emocional dos cuidadores de idosos. Fizeram parte deste estudo, 124 cuidadores de idosos. O levantamento de dados foi realizado no período de janeiro a julho de 2009. Para a obtenção dos dados, foram utilizados instrumentos para caracterização do cuidador, em relação a sexo, idade, estado civil, grau de parentesco, conhecimento sobre a doença, a realização de curso formal, horas dedicadas ao cuidado, atividades do cuidador e apoio recebido. Também foram utilizadas a Escala de Sobrecarga de Zarit e Self-Reporting Questionaire (SRQ-20) para avaliar o impacto percebido pelo ato de cuidar sobre a saúde física e emocional do cuidador e a detecção de desconforto emocional, respectivamente. Os resultados encontrados foram que $85,6 \%$ dos cuidadores são do sexo feminino, com 56 anos de idade em média, utilizam aproximadamente 12,4 horas diárias para o cuidado com o idoso, que 57,56\% das pessoas estudadas apresentaram grau de sobrecarga leve a moderada e que a sobrecarga é fator de risco para o desconforto emocional, sobretudo para cuidadores com mais de 60 anos e do sexo feminino.

A realização de um estudo transversal descritivo e analítico feito por Cruz, Lecheta e Waccholz (2009) teve por objetivo investigar os fatores associados à depressão e à sobrecarga nos cuidadores de idosos com a doença de Alzheimer atendidos na Unidade de Atenção ao Idoso de Curitiba-PR. Os sintomas depressivos foram avaliados através das Escalas de Depressão CES-D e a sobrecarga no cuidador foi avaliada pela escala Zarit Burden Interview (ZBI), além do uso de protocolo para coleta de dados dos participantes. Os resultados demonstram que $81 \%$ dos cuidadores são do sexo feminino, com média de 53 anos de idade. As principais variáveis associadas à depressão e à sobrecarga se devem a qualidade da relação pré-mórbida, o fato de ser mulher, a prática de atividades físicas, participação em atividades sociais e o uso de psicotrópicos.

O estudo realizado por Gaioli, Furegato e Santos (2012), descreveu as variáveis sociodemográficas e de saúde e associá-las à resiliência de cuidadores de idosos com a doença de Alzheimer. Foram participantes 101 cuidadores acompanhantes de idosos de unidades básicas e de um hospital público, que responderam um questionário social, Inventário de Depressão de Beck e Escala de Resiliência. Por resultados encontraram maior participação de cuidadores do sexo feminino, recebiam ajuda de outras pessoas e possuíam alto grau de resiliência. A maioria dos cuidadores era da própria família, sem atividade profissional remunerada, sem experiência e sem a realização de curso para preparação adequada. Também foi encontrada a presença de cuidadores idosos com mais de 60 anos, aproximadamente $40 \%$ dos cuidadores. Tal resultado demonstra o desafio de lidar com a saúde dos cuidadores, uma vez que esses também estão envelhecendo. Nenhum dos cuidadores atingiu escore para a depressão. A resiliência apresentou associação significativa com as variáveis grau de parentesco, tratamento médico, uso de medicamento, cansaço, esgotamento, desânimo e saúde mental do cuidador. 
Stackfleth et al. (2012) realizaram um estudo transversal, com 60 cuidadores cujo objetivo foi avaliar a sobrecarga dos cuidadores de idosos fragilizados que residem em domicílios. Os participantes tinham idade igual ou acima de 60 anos, ambos os sexos, cuidadores de idosos considerados frágeis e residentes no mesmo local. A coleta de dados foi realizada entre os meses de novembro/2010 e fevereiro/2011. Na primeira etapa da pesquisa, realizada para delimitar a amostra de cuidadores de idosos frágeis, utilizou-se um inventário sociodemográfico, a Edmonton Frail Scale para avaliar a fragilidade dos idosos e a Escala de Medida de Independência Funcional (MIF) para avaliar a independência funcional. Já na segunda etapa, realizado o levantamento de dados para se investigar a sobrecarga, foi utilizada a Zarit Burden Interview (ZBI), que avalia a sobrecarga dos cuidadores, associada à capacidade funcional dos pacientes, a seus distúrbios de comportamento e às situações quotidianas. Como resultado, observou-se que a maioria dos cuidadores era do sexo feminino (75\%), casadas $(58,3 \%)$ e $45 \%$ eram filhos. Evidenciou-se correlação positiva e moderada entre o grau de dependência funcional do idoso e a sobrecarga do cuidador. Quanto à escolaridade, $80 \%$ dos cuidadores afirmaram ter de 5 a 8 anos de estudo. $\mathrm{O}$ alto nível de sobrecarga foi demonstrada em $8 \%$ dos cuidadores, obtendo escores igual ou superior avaliado na ZBI.

Considerando o exposto nos estudos relatados, a hipótese para esta pesquisa é que o cuidador, por estar ligado afetivamente ao idoso dependente, pode acabar por dedicar-se pouco tempo a si mesmo, resultando em um sentimento de menos valia com relação aos cuidados com a própria saúde biopsicossocial, levando-o a apresentar sintomas depressivos. Este estudo tratou-se de uma pesquisa de levantamento que teve por objetivo rastrear a sintomatologia depressiva em cuidadores informais de idosos com limitações para a realização de atividades de vida diária, além de consideras diferenças quanto às variáveis sexo, idade, grau de parentesco, escolaridade, residência, situação profissional, renda familiar, tempo diário para o cuidado, presença de segundo cuidador e da realização de curso formal para os cuidados.

\section{METODOLOGIA}

\section{Participantes}

Foram participantes deste estudo 46 cuidadores informais de idosos, sendo 35 (76,1\%) do sexo feminino e $11(23,9 \%)$ do sexo masculino. A idade média dos cuidadores foi de 46,89 anos $(\mathrm{DP}=13,91)$, variando entre 22 e 79 anos. Dentre os 46 participantes, $38(82,6 \%)$ têm idade até 59 anos e $8(17,4 \%)$ possuem idade igual ou acima de 60 anos. Em relação ao grau de parentesco dos participantes, os resultados encontrados estão dispostos na Tabela 1 , indicando que a maioria foi composta por filhos (as) $(56,5 \% ; n=26)$, seguido por cônjuges $(10,9 \% ; n=5)$ e noras $(10,9 \% ; n=5)$ dos idosos.

Tabela 1 - Distribuição dos participantes em relação ao grau de parentesco

\begin{tabular}{ccc}
\hline Parentesco & $\mathbf{N}$ & $\mathbf{\%}$ \\
\hline Filho (a) & 26 & 56,5 \\
Cônjuge & 05 & 10,9 \\
Noras & 05 & 10,9 \\
Netos (as) & 03 & 6,5 \\
Irmãos (ãs) & 02 & 4,3 \\
Outros & 05 & 10,9 \\
\hline Total & 46 & 100 \\
\hline \multicolumn{2}{c}{ Fonte: Próprios autores }
\end{tabular}


Em relação à variável escolaridade, a maioria dos cuidadores possuíam o ensino fundamental incompleto ou ensino médio completo, ambos com 34,8\% ( $n=16)$, conforme apresentado na Tabela 2 . No que se refere à residência 71,7\% $(n=33)$ afirmaram que residiam no mesmo domicílio do idoso e 28,3\% $(n=13)$ residiam em casas separadas. Já em relação à situação profissional $45,7 \%(n=21)$ trabalhava, 39,1\% $(n=18)$ estava desempregada e $15,2 \%$ $(n=7)$ estava aposentada. Quanto à renda familiar 54,3\% $(n=25)$ declararam que estava entre 1 a 3 salários mínimos, 43,5\% $(n=20)$ acima de 3 salários mínimos e 2,2\% $(n=1)$ tinha renda familiar de até 1 salário mínimo.

Tabela 2 - Distribuição dos participantes em relação à escolaridade

\begin{tabular}{ccc}
\hline Escolaridade & $\mathbf{N}$ & $\mathbf{\%}$ \\
\hline Analfabeto & - & - \\
Ensino Fundamental Incompleto & 16 & 34,8 \\
Ensino Fundamental Completo & 5 & 10,9 \\
Ensino Médio Incompleto & 1 & 2,2 \\
Ensino Médio Completo & 16 & 34,8 \\
Ensino Superior ou Mais & 8 & 17,4 \\
\hline Total & 46 & 100 \\
\hline Fonte: Próprios autores & &
\end{tabular}

Levando-se em conta a variável tempo diário para o cuidado prestado ao idoso, $50 \%$ $(n=23)$ dos participantes relataram dedicação total aos cuidados necessários, conforme demonstrado na Tabela 3. Em relação à divisão das responsabilidades de cuidar do idoso (quando há um segundo cuidador), a maioria dos participantes $(54,3 \% ; n=25)$ informou que não há outra pessoa para auxiliar nos cuidados ao idoso e $(45,7 \% ; n=21)$ indicaram receber ajuda de outras pessoas. Ainda, é importante mencionar que nenhum dos cuidadores relatou ter realizado curso formal para as práticas de cuidados básicos do idoso.

Tabela 3 - Tempo diário para o cuidado ao idoso

\begin{tabular}{ccc}
\hline Horas Diárias & N & \% \\
\hline De 1 a 3 horas & 8 & 17,4 \\
De 3 a 6 horas & 8 & 17,4 \\
De 6 a 9 horas & 7 & 15,2 \\
Acima de 9 horas & 23 & 50,0 \\
\hline Total & 46 & 100 \\
\hline \multicolumn{2}{c}{ Fonte: Próprios autores }
\end{tabular}

\section{Instrumentos}

Inventário Sociodemográfico do Cuidador Informal: elaborado para identificar características sociodemográficas, como estado civil, sexo, escolaridade, grau de parentesco do (a) idoso (a), tempo dedicado ao cuidado e tipo de dependência.

Escala Baptista de Depressão - Versão Adulto- EBADEP-A (BAPTISTA, 2012): tem por objetivo rastrear sintomas da depressão em amostras psiquiátricas e não psiquiátricas. É composta por 90 frases, dispostas em pares (um pólo negativo e outro positivo), totalizando 45 itens, respondidos em uma escala do tipo Likert de quatro pontos (de zero a três), tendo como pontuação mínima zero e máxima de 135 pontos. Quanto menor a pontuação apresentada na escala, menor a sintomatologia depressiva apresentada pelo respondente. Apresenta características psicométricas adequadas de confiabilidade teste-reteste, validade de 
construto, critério, confiabilidade e sensibilidade/ especificidade do instrumento (BAPTISTA; CARDOSO; GOMES; 2012)

\section{Procedimento}

Após a aprovação do Comitê de Ética em Pesquisa da Universidade São Francisco, da cidade de Campinas-SP (CAAE 38626814.4.0000.5514), realizou-se os agendamentos para as aplicações, considerando a disponibilidade da instituição e dos participantes. A coleta de dados aconteceu de forma individual. Após o esclarecimento dos objetivos da pesquisa e assinatura dos participantes do Termo de Consentimento Livre e Esclarecido, os instrumentos foram entregues. O tempo aproximado para a resposta dos instrumentos foi de 30 minutos.

\section{RESULTADOS E DISCUSSÃO}

Os dados coletados por meio da EBADEP-A foram analisados pela estatística descritiva considerando o grupo de participantes em seu total e também divididos de acordo sexo, idade, residência, grau de parentesco, escolaridade, situação profissional, renda, tempo de atenção ao idoso, presença do segundo cuidador e conclusão de curso formal para cuidadores, estabelecido pelos dados obtidos com o Inventário Sociodemográfico do Cuidador Informal. Também foram analisados por meio da estatística inferencial (teste $t$ de Student e ANOVA).

No que se refere aos resultados encontrados na EBADEP-A, apresentados na Tabela 4, a média total foi de 37,09, indicando não existir sintomatologia depressiva nos participantes. O item 42 (Meu desejo sexual vem diminuindo muito) foi o que apresentou a maior média, seguido pelos itens 14 (Está mais difícil decidir), 38 (Ando mais cansado), 37 (Qualquer coisa me deixa nervoso) e 36 (Perco a paciência por muito pouco). Tais resultados podem ser justificados pelo fato do avançar da idade dos cuidadores e da sobrecarga que o cuidado acarreta, o que possivelmente dificultaria no controle das próprias emoções (PEREIRA; CARVALHO, 2012; TOMOMITSU; PERRACINI; NERI, 2013). As médias mais baixas se deram nos itens 44 (Gosto de tomar remédio por precaução) 32 (Não consigo mais fazer o necessário), 33 (Não dou mais valor à minha vida) 34 (Não gosto mais de mim), 29 (Tenho pensado que seria melhor estar morto) e 20 (As coisas que faço não ajudam a mais ninguém). Rocha, Vieira e Sena (2008) e Almeida et al (2013) ressaltam que o cuidar pode trazer consequências positivas, uma vez que as pessoas podem encontrar nessa tarefa um sentido na vida, uma oportunidade de exercer a espiritualidade e uma forma de encontrar o equilíbrio biopsicossocial ao cuidar do idoso com carinho.

O teste $t$ de Student foi realizado para verificar se existiam diferenças estatisticamente significativas na EBADEP-A em relação ao sexo dos cuidadores. Os participantes do sexo feminino apresentaram média de 39,37 $(D P=23,78)$, já os participantes do sexo masculino obtiveram média de 29,82 ( $D P=16,74)$. Porém, o teste $t$ de Student não demonstrou diferença significativa entre os grupos $(t[23,89]=1,48 ; p=0,152)$. Ainda que não tenha sido verificada diferença entre os sexos, os achados reforçam o papel social da mulher como provedora de cuidados indicada pela prevalência de cuidadores do sexo feminino. Esse contexto também foi observado em estudos anteriores (PESSANHA, 2013; GRATAO et al. 2012). Pessanha (2013) afirma que mesmo diante de todos os desafios da mulher no contexto social ela ainda assume o papel de provedora de cuidados. Gratao et al. (2012) salientam que a mulher prejudica seu tempo livre de lazer e vida social e acumula atividades laborais e domésticas para atender as necessidades do idoso, gerando uma sobrecarga física e emocional.

Quanto à idade, foram considerados dois grupos, sendo o primeiro composto por participantes categorizados como idosos (60 anos ou mais) e o segundo com as pessoas não idosas de acordo com a classificação proposta pela OMS (2005). Os participantes do primeiro 
grupo apresentaram média de 37,97 $(D P=23,89)$, já os participantes do segundo grupo obtiveram média de 32,88 $(D P=14,62)$. O teste $t$ de Student não indicou diferença estatisticamente significativa entre os grupos $(t[16,118]=0,789 ; p=0,442)$. É interessante destacar que 17,4\% $(n=8)$ são idosos cuidadores de idosos. O estudo realizado por Pawlowski et al. (2010) não corrobora com os resultados aqui encontrados, posto que verificaram níveis maiores de depressão em cuidadores da faixa etária entre 56 e 64 anos quando comparados a não cuidadores. Fortes-Burgos, Neri e Cupertino (2008, apud PAWLOWSKI et al., 2010) também encontraram associação de maior risco de ocorrência de depressão na faixa etária de 60 a 69 anos. Camarano e Kanso (2010, apud TOMOMITSU; PERRACINI; NERI, 2013) afirmam que há tendência futura para o aumento de idosos cuidando de pessoas muito idosas no Brasil e uma pesquisa realizada com cuidadores de idosos indicou que 40\% desses participantes eram idosos (GAIOLI; FUREGATO; SANTOS, 2012) sendo que, futuramente, o grande desafio das famílias será lidar com as doenças que acometerem os cuidadores, já que estes passam boa parte do tempo sozinhos com os idosos e precisarão de cuidados decorrentes de suas próprias limitações, o que podem prejudicar o desempenho das atividades de cuidado (GAIOLI; FUREGATO; SANTOS, 2012).

No que se referiu à residência, a média da EBADEP-A em relação àqueles que moram com o idoso foi de 42,58 ( $D P=20,31)$ e dos que não moram com o idoso foi igual a 25,69 $(D P=24,57)$. O teste $t$ de Student indicou diferença marginalmente significativa entre os grupos $(t[18,813]=2,069 ; p=0,053)$. Assim verifica-se neste estudo que, em média, os cuidadores que moravam com o idoso apresentaram maior sintomatologia depressiva do que os que não moram com o idoso. Residir na mesma casa pode fazer com que o cuidador direcione grande parte do tempo aos cuidados necessários ao idoso, sem que tenha um horário para o autocuidado físico e psicológico, ou ainda, pode ter a qualidade de vida prejudicada por conta da sobrecarga emocional e física (PEREIRA; CARVALHO, 2012). Uma característica desta amostra é que a maioria dos entrevistados, além de ser cuidador, possui parentesco próximo ao idoso, o que pode caracterizar o maior envolvimento emocional. Pesquisa realizada por Pessanha (2013) encontrou que 90\% dos cuidadores também residiam com o idoso cuidado. Também os resultados de Gaioli et al. (2012) mostram que a maioria dos cuidadores participantes da pesquisa $(55,4 \%)$ residiam com o idoso.

Quanto ao grau de parentesco, em razão de não haver variabilidade suficiente em algumas categorias, foi necessário reorganizá-las, considerando o grupo de cônjuges, filhos, noras e outros. Os filhos obtiveram a maior média dentre todos os grupos, seguido do grupo de cônjuges. Apesar disso, a ANOVA não indicou diferença estatisticamente significativa para a variável parentesco $(F[3,42]=1,660 ; p=0,190)$. O resultado é semelhante ao proposto por Pawlowski et al. (2010), que também encontraram uma maior sobrecarga psicológica entre os filhos que exercem o papel de cuidador. Geralmente, os filhos relatam que assumiram a responsabilidade de cuidador em decorrência do sentimento de retribuição e gratidão. O que leva um familiar ao exercício da tarefa de cuidar são sentimentos de amor, nível de afinidade com o idoso, carinho, afeto, culpa, dever ou por não haver outra possiblidade e demandam um novo arranjo familiar (GIL; BERTUZZI, 2006; PESSANHA, 2013).

Também foi preciso reorganizar a variável escolaridade considerando o grupo de ensino fundamental incompleto, ensino fundamental completo, ensino médio completo e ensino superior ou mais. A média maior foi apresentada pelo grupo com escolaridade de ensino fundamental completo $(M=52,00)$, seguido de ensino médio completo $(M=36,94)$, ensino superior ou mais $(M=35,00)$ e ensino fundamental incompleto $(M=32,69)$. Por meio da ANOVA não foi verificada diferença estatisticamente significativa entre os grupos $(F[3,42]=1,113 ; p=0,354)$. Em outros estudos sobre cuidadores informais de idosos também não foram encontradas diferenças estatisticamente significativas quanto ao grau de escolaridade (GRATÃO et al., 2012; PAWLOWSKI et.al., 2010). Pessanha (2013) afirma que é importante conhecer o grau de escolaridade dos cuidadores, pois são esses que são 
orientados quanto aos cuidados e há atividades que demandam certa compreensão para serem realizadas de forma adequada, como exemplo, a administração de medicamentos.

Tabela 4 - Frequência de respostas e médias por item da EBADEP-A

\begin{tabular}{|c|c|c|c|c|}
\hline Itens EBADEP-A & Mínimo & Máximo & Media & $\mathrm{DP}$ \\
\hline 1 & 0 & 3 & 1,07 & 1,041 \\
\hline 2 & 0 & 3 & 1,24 & 0,947 \\
\hline 3 & 0 & 3 & 0,67 & 0,896 \\
\hline 4 & 0 & 3 & 0,59 & 0,832 \\
\hline 5 & 0 & 3 & 0,96 & 1,115 \\
\hline 6 & 0 & 3 & 1,07 & 1,083 \\
\hline 7 & 0 & 3 & 0,72 & 0,981 \\
\hline 8 & 0 & 3 & 0,52 & 0,752 \\
\hline 9 & 0 & 3 & 0,65 & 0,795 \\
\hline 10 & 0 & 3 & 0,67 & 0,92 \\
\hline 11 & 0 & 3 & 0,74 & 0,953 \\
\hline 12 & 0 & 3 & 0,85 & 1,115 \\
\hline 13 & 0 & 3 & 0,50 & 0,81 \\
\hline 14 & 0 & 3 & 1,41 & 1,066 \\
\hline 15 & 0 & 3 & 0,80 & 0,91 \\
\hline 16 & 0 & 3 & 1,00 & 1,095 \\
\hline 17 & 0 & 3 & 0,96 & 1,074 \\
\hline 18 & 0 & 3 & 1,13 & 1,222 \\
\hline 19 & 0 & 3 & 1,02 & 1,000 \\
\hline 20 & 0 & 2 & 0,28 & 0,502 \\
\hline 21 & 0 & 3 & 0,43 & 0,860 \\
\hline 22 & 0 & 3 & 0,87 & 1,147 \\
\hline 23 & 0 & 3 & 0,78 & 0,964 \\
\hline 24 & 0 & 3 & 1,09 & 1,029 \\
\hline 25 & 0 & 3 & 1,17 & 1,060 \\
\hline 26 & 0 & 3 & 0,74 & 0,828 \\
\hline 27 & 0 & 3 & 1,09 & 1,112 \\
\hline 28 & 0 & 2 & 0,46 & 0,622 \\
\hline 29 & 0 & 3 & 0,35 & 0,766 \\
\hline 30 & 0 & 3 & 0,43 & 0,779 \\
\hline 31 & 0 & 3 & 0,93 & 1,083 \\
\hline 32 & 0 & 2 & 0,39 & 0,577 \\
\hline 33 & 0 & 2 & 0,39 & 0,614 \\
\hline 34 & 0 & 2 & 0,35 & 0,604 \\
\hline 35 & 0 & 2 & 0,63 & 0,679 \\
\hline 36 & 0 & 3 & 1,33 & 0,990 \\
\hline 37 & 0 & 3 & 1,35 & 1,016 \\
\hline 38 & 0 & 3 & 1,37 & 1,162 \\
\hline 39 & 0 & 3 & 0,98 & 0,977 \\
\hline 40 & 0 & 3 & 0,67 & 0,944 \\
\hline 41 & 0 & 3 & 0,96 & 1,154 \\
\hline 42 & 0 & 3 & 1,52 & 1,188 \\
\hline 43 & 0 & 3 & 0,80 & 0,980 \\
\hline 44 & 0 & 2 & 0,22 & 0,467 \\
\hline 45 & 0 & 3 & 0,93 & 1,124 \\
\hline EBADEP Total & 0 & 96 & 37,09 & 22,506 \\
\hline
\end{tabular}

Fonte: Próprios autores

No concernente à situação profissional, verificou-se diferença significativa no resultado da ANOVA $(F[2,43]=6,30 ; p=0,01)$. O teste de Tukey Post Hoc, disposto na Tabela 5, demonstrou a existência de dois grupos. O primeiro composto por aposentados $(M=$ $25,29)$ e os que estavam empregados $(M=29,81)$ e o segundo grupo composto por pessoas desempregadas $(M=50,17)$, com maiores médias que o primeiro grupo. Na amostra estudada, os sintomas depressivos aparecem de maneira mais preponderantes no grupo de 
desempregados. Não se pode afirmar que o fato do indivíduo ser cuidador de uma pessoa idosa seja a única variável responsável pela sintomatologia depressiva uma vez que a história de vida, sentimento de sobrecarga e dever, construção de afeto com o paciente, entre outros fatores podem influenciar na maneira como o sujeito percebe a si mesmo, aos demais e ao mundo. Os resultados do estudo realizado por Gaioli, Furegato e Santos (2012) apontam que a impossibilidade de realizar atividade profissional remunerada, de lazer e de vida social em decorrência dos cuidados dispensados ao idoso gera sobrecarga emocional, problemas de saúde e estresse, bem como de insegurança e falta de processos de autocuidados.

Tabela 5 - Teste de Tukey Post Hoc considerando a situação profissional

\begin{tabular}{cccc}
\hline Situação Profissional & N & \multicolumn{2}{c}{$\alpha=0,05$} \\
\cline { 3 - 4 } & & 1 & 2 \\
\hline Aposentados & 7 & 25,29 & \\
Empregados & 21 & 29,81 & \\
Desempregados & 18 & & 50,17 \\
\hline$p$ & & 0,846 & 1,000 \\
\hline Fonte: Próprios autores & &
\end{tabular}

Considerando a variável renda familiar, foram considerados três grupos, sendo os que ganham menos de um salário mínimo, entre um e três salários mínimos e acima de três salários mínimos. Porém para análise só foram utilizados os dois últimos porque o primeiro grupo apresentou apenas um participante. Os participantes do grupo com renda familiar entre um e três salários mínimos tiveram média de 36,12 ( $D P=20,01)$, já aqueles com renda familiar maior que três salários tiveram média de 40,00 $(D P=24,95)$, não sendo evidenciadas diferenças significativas pelo teste $t$ de Student $(t[36,03]=-0,56 ; \mathrm{p}=0,576)$. Os achados encontrados precisam ser explorados com maior profundidade, uma vez que alguns autores corroboram com o presente estudo (Pawlowski et al., 2010) e outros não (TOMOMITSU; PERRACINI; NERI, 2013). Pawlowski et al. (2010) não encontraram diferença significativa para a variável renda quando analisada a depressão. Todavia, Tomomitsu, Perracini e Neri (2013) encontraram associações entre a baixa renda e a presença de sintomas depressivos e menor nível de satisfação com a vida, principalmente ao se considerar cuidadores sem suporte social adequado.

O tempo utilizado para o cuidado diário do idoso foi analisado por meio da ANOVA. Foram considerados grupos que dedicam cuidado entre uma a três horas $(M=36,88)$, três e seis horas $(M=20,38)$, de seis a nove horas $(M=34,57)$ e acima de nove horas $(M=43,74)$. Não foi identificada diferença estatisticamente significativa entre os grupos $(F[3,42]=2,367$; $p=0,084)$. Entretanto, Gratão et al. (2012) reforçam que o sentimento de sobrecarga, esgotamento e exaustão são resultantes de grande dedicação na realização da atividade de cuidar e diante desta necessidade o cuidador desconsidera suas próprias necessidades pessoais. Os autores ainda afirmam que tal situação é um fator de risco para o desconforto emocional, como tristeza e ansiedade, sendo comum a presença de cuidadores depressivos quando expostos a um período de dedicação prolongado. Ainda, de acordo com Pereira e Carvalho (2012), a sobrecarga emocional e física, bem como a ansiedade e a depressão estão associadas negativamente com a qualidade de vida.

Em relação à divisão de tarefas com o segundo cuidador, verificou-se média de 36,86 $(D P=28,44)$ para o grupo que não possui o auxílio de outro cuidador e média $37,28(D P=$ $16,60)$ para o grupo que recebe ajuda de outros cuidadores. Considerando o teste $t$ de Student não foi evidenciada diferença estatisticamente significativa em relação a tal variável $(t[44]=-$ 0,06; $p=0,950$ ). Pessanha (2013) destaca que o segundo cuidador normalmente é um parente próximo, residente na mesma casa ou não e que possui um nível de afetividade próximo com 
o idoso, ao passo que Pereira e Carvalho (2012) ressaltam que um cuidador secundário pode contribuir com uma maior qualidade de vida e satisfação conjugal de cuidadores primários. Observa-se, apesar dos resultados da presente investigação, a necessidade de implementar meios para o suporte adequado aos cuidadores não assistidos por outras pessoas de seu convívio, já que há grande ausência de políticas públicas de saúde e de apoio social para esta população (PESSANHA, 2013).

Não houve a realização de curso formal para os cuidados dispensados ao idoso por nenhum dos participantes desta pesquisa. $\mathrm{O}$ fato de não haver preparo adequado a respeito do cuidado e de esclarecimentos sobre as necessidades do idoso, doença, evolução e tratamento, pode acarretar risco e/ ou prejuízo nas mais diversas situações de cuidado ao idoso (GAIOLI; FUREGATO; SANTOS, 2012). Participar de grupos de apoio, possibilitando o fortalecimento psíquico e social, pode contribuir para o autocuidado, planejamento de vida e para reinvindicação de direitos assistenciais e sociais, sendo alternativa para orientação ao cuidador.

\section{CONCLUSÃO}

A população mundial vem envelhecendo ao longo dos anos e o Brasil também tem vivenciado este crescimento demográfico. Esta situação se relaciona ao aumento de idosos com limitações para a realização de atividades de vida diária que precisam receber cuidados de outras pessoas.

Esta pesquisa alcançou o objetivo a que se propôs ao contribuir com o aumento de conhecimento sobre a presença de sintomatologia depressiva vivenciada por cuidadores informais de idosos. Conhecer os aspectos psicológicos do cuidador torna-se importante para o desenvolvimento de ações voltadas ao fortalecimento da sua saúde mental e de melhoria da qualidade de vida tanto do cuidador quanto do idoso. Ao se traçar um perfil de cuidadores tem-se subsídios para o planejamento de ações sociais e políticas públicas a fim de assegurar melhores condições econômicas, de acesso à saúde e bem-estar psicológico de modo a proporcionar cuidados de boa qualidade ao idoso com limitações.

Há de se considerar as limitações do presente estudo para se ponderar o alcance dos resultados encontrados, tais como, o número da amostra e região de residência dos participantes, o que a torna homogênea e não generalizável para a população geral. Além disso, sugere-se a realização de novos estudos que façam aplicação de outros instrumentos que avaliem outros impactos psicológicos nos cuidadores, com amostras mais representativas e de forma a explorar melhor o contexto no qual o idoso e cuidador estão inseridos, especialmente quando não houver grau de parentesco entre eles.

\section{REFERÊNCIAS}

ALMEIDA, S.S.L.; MARTINS, A.M.; REZENDE, A.M.; SCHALL, V.T.; MODENA, C.M. Sentidos do cuidado: a perspectiva de cuidadores de homens com câncer. PsicoUSF, Itatiba, v. 18, n. 3, p. 469-478. set./dez. 2013.

APA. Manual diagnóstico e estatístico de transtornos mentais. 5. ed. Porto Alegre: Artmed, 2014.

BAPTISTA, M.N. Suicídio e depressão: atualizações. Rio de Janeiro: Guanabara Koogan, 2004. 
Paulo: Vetor, 2012.

BAPTISTA, M.N.; CARDOSO, H.F.; GOMES, J.O. Escala Baptista de depressão (Versão Adulto) - EBADEP-A: validade convergente e estabilidade temporal. Psico-USF, Itatiba, v. 17, n. 3, p. 407-416, set./dez. 2012.

BAPTISTA, M.N.; GOMES, J.O. Escala Baptista de depressão (Versão Adulto)EBADEP-A: evidências de validade de construto e de critério. Psico-USF, Itatiba, v. 16, n. 2, p. 151-161, mai./ago. 2011.

BAPTISTA, M.N.; MORAIS, P.R.; RODRIGUES, T.; SILVA, J.A.C. Correlação entre sintomatologia depressiva e prática de atividades sociais em idosos. Avaliação Psicológica, Porto Alegre, v. 5, n. 1, p. 77-85, jun. 2006.

BODSTEIN, A.; LIMA, V.V.A.; BARROS, A.M.A. A vulnerabilidade do idoso em situações de desastres: necessidade de uma política de resiliência eficaz. Ambiente \& Sociedade, São Paulo, v. 17, n. 2, p. 157-174, abr./jun. 2014.

COUTINHO, M.P.L.; GONTIÈS, B.; ARAUJO, L.F.; SÁ, R.C.N. Depressão, um sofrimento sem fronteiras: representações sociais entre crianças e idosos. Psico$U S F$, Itatiba, v. 8, n. 2, p. 183-192, jul./dez. 2003.

CRUZ, M.N.; LECHETA, D.R.; WACHHOLZ, P.A. Fatores associados à sobrecarga e à depressão em cuidadores de idosos com doença de Alzheimer. Revista Brasileira de Geriatria \& Gerontologia, Rio de Janeiro, v. 3, n. 1, p. 15-23, ago. 2009.

DIAS JUNIOR, C.S.; COSTA, C.S; LACERDA, M.A. O envelhecimento da população brasileira: uma análise de conteúdo das páginas da REBEP. Revista Brasileira de Geriatria e Gerontologia, Rio de Janeiro, v. 9, n. 2, p. 7-24. ago. 2006.

FERREIRA, H.G.; LIMA, D.M.X.S; ZERBINATTI, R. Atendimento psicoterapêutico cognitivo-comportamental em grupo para idosos depressivos: um relato de experiência. Revista da SPAGESP, Ribeirão Preto, v. 13, n. 2, p. 86-101. 2012.

GAIOLI, C.C.L.O;; FUREGATO, A.R.F; SANTOS, J.L.F. Perfil de cuidadores de idosos com doença de Alzheimer associado à resiliência. Texto \& Contexto Enfermagem,Florianópolis, v. 21, n. 1, p. 150-157. jan./mar. 2012.

GIL, M.E.; BERTUZZI, L.D. Desafios para a psicologia no cuidado com o cuidador. Revista Bioética, v. 14, n. 1, p. 49-59. 2006.

GRATAO, A.C.M.; VENDRUSCOLO, T.R.P.; TALMELLI, L.F.S.; FIGUEIREDO, L.C.; SANTOS, J.L.F; RODRIGUES, R.A.P. Sobrecarga e desconforto emocional em cuidadores de idosos. Texto \& Contexto - Enfermagem, Florianópolis, v. 21, n. 2, p. 304-312. abr./jun. 2012.

MENDES, M.R.S.S.B.; GUSMÃO J.L.; FARO, A.C.M; LEITE, R.C.B.O. A situação social do idoso no Brasil: uma breve consideração. Acta Paulista de Enfermagem, São Paulo, v. 18, n. 4, p. 422-426. out./dez. 2005. 
MTE. Classificação Brasileira de Ocupações: CBO 2002. Brasília: TEM, SPPE, 2002.

OMS. Envelhecimento ativo: uma política de saúde. Brasília: Organização Pan-Americana da Saúde, 2005.

PAPALIA, D.E.; OLDS, S.W.; FELDMAN, R.D. Desenvolvimento humano. 8. ed. Porto Alegre: Artmed, 2006.

PAWLOWSKI, J.; GONÇALVES, T.R.; HILGERT, J.B.; HUGO, F.N.; BOZZETTI, M.C; BANDEIRA, D.R.. Depressão e relação com a idade em cuidadores de familiares portadores de síndrome demencial. Estudos de Psicologia, Rio Grande do Norte, v. 15, n. 2, p. 173-180. mai./ago. 2010.

PEREIRA, M.G.; CARVALHO, H. Qualidade vida, sobrecarga, suporte social, ajustamento conjugal e morbidade psicológica em cuidadores de idosos com dependência funcional. Temas em Psicologia, Ribeirão Preto, v. 20, n. 2, p. 369-383. 2012.

PESSANHA, A.F. O fardo em cuidadores informais de pacientes dementados. Dissertação (Mestrado em Gerontologia), Programa de Pós-Graduação Stricto-Sensu em Gerontologia, Pontifícia Universidade Católica de Brasília, Brasília. 2013.

ROCHA, M.P.F.; VIEIRA, M.A.; SENA, R.R. Desvelando o cotidiano dos cuidadores informais de idosos. Revista Brasileira de Enfermagem, Brasília, v. 61, n.6, p. 801808. nov./dez. 2008.

SILVA, M.C. O processo de envelhecimento no Brasil: desafios e perspectivas. Textos Sobre Envelhecimento, Rio de Janeiro, v. 8, n. 1, p. 43-60. 2005.

SILVEIRA, T.M.; CALDAS, C.P.; CARNEIRO, T.P. Cuidando de idosos altamente dependentes na comunidade: um estudo sobre cuidadores familiares principais. Caderno de Saúde Pública, Rio de Janeiro, v. 22, n. 8, p. 1629-1638. ago. 2006.

SOMMERHALDER, C. (2001). Significados associados à tarefa de cuidar de idosos de alta dependência no contexto familiar. 106 f. Dissertação (Mestrado em Educação) Faculdade de Educação, Universidade Estadual de Campinas, Campinas. 2001.

STACKFLETH, R.; DINIZ, M.A., FHON, J.R.S.; VENDRUSCOLO, T.R., FABRÍCIOWHEBE, S.C.C.; MARQUES, S.; RODRIGUES, R.A.P. Sobrecarga de trabalho em cuidadores de idosos fragilizados que vivem no domicilio. Acta Paulista de Enfermagem, São Paulo, v. 25, n. 5, p. 768-774. 2012.

TOMOMITSU, M.R.S.V.; PERRACINI, M.R; NERI, A.L. Influência de Gênero, Idade e Renda Sobre o Bem-Estar de Idosos Cuidadores e Não Cuidadores. Revista Brasileira de Geriatria e Gerontologia, Rio de Janeiro, v. 16, n. 4, p. 663-680. 2013. 